\title{
ESTUDO DA SIMILARIDADE ENTRE ESCALARES SOBRE UMA SUPERFÍCIE HETEROGÊNEA UTILIZANDO LARGE-EDDY SIMULATION
}

\author{
Diana Maria Cancelli ${ }^{1}$, Marcelo Chamecki ${ }^{2}$, Nelson Luis Dias ${ }^{3}$ \\ ${ }^{1}$ Programa de Pós-Graduação em Métodos Numéricos em Engenharia, UFPR - Brasil; \\ ${ }^{2}$ Department of Meteorology, Pennsylvania State University - USA; \\ ${ }^{3}$ Departamento de Engenharia Ambiental, UFPR - Brasil
}

\section{RESUMO}

A similaridade entre flutuações de escalares na camada limite atmosférica (CLA) é hipótese fundamental em estudos relacionados com poluição do ar, disponibilidade hídrica e emissão de gases de efeito estufa. Em função de sua importância e da dificuldade de verificação de sua validade a partir de experimentos de campo, neste trabalho utilizamos Large-eddy Simulation (LES) para tentar entender o comportamento das flutuações de temperatura e umidade específica sobre uma superfície heterogênea.

\begin{abstract}
In the atmospheric boundary layer (ABL), the similarity between scalar fluctuations is a fundamental hypothesis in studies related to air pollution, hydrology, and emission of greenhouse gases. Because of its importance, and the difficult in verifying its validity using data from field experiments, in this work we use Large-Eddy Simulations (LES) as a tool to understand the behavior of the temperature and specific humidity fluctuations over a heterogenous surface.
\end{abstract}

\section{INTRODUÇÃO}

Uma hipótese fundamental da Teoria de Similaridade de Monin-Obukhov é que as flutuações de dois escalares quaisquer possuem comportamento similar na camada limite superficial (CLS), isto é, todas as funções de similaridade de Monin-Obukhov para os dois escalares são iguais; existe, no entanto, um grande debate em torno da validade desta hipótese e das possíveis causas de suas violações (referências podem ser encontradas em Cancelli et al., 2012). Dentre as causas de violação da hipótese de similaridade está a heterogeneidade da superfície; em experimentos de campo é difícil separar os efeitos desta de outros como advecção local e entranhamento no topo da CLA. Com o objetivo de avaliar os efeitos da heterogeneidade da superfície sobre o comportamento do coeficiente de correlação entre temperatura e umidade específica (um dos principais indicadores de similaridade entre escalares na CLA), foram realizadas simulações de LES.

As principais informações relacionadas ao modelo de LES e às simulações realizadas são apresentadas na sequência.

\section{O MODELO DE LES}

A base do código de LES utilizado no desenvolvimento deste trabalho está descrita em Kumar et al. (2006). Basicamente, para um escoamento incompressível, são resolvidas as 
equações de Navier-Stokes tridimensionais para transporte de massa, momentum e de conservação de escalares - neste trabalho, os escalares simulados foram temperatura e umidade específica.

\section{SIMULAÇÕES}

Para todas as simulações foi utilizada uma grade com $128^{3}$ pontos, com resolução de 40x40x $16 \mathrm{~m}^{3}$, resultando num domínio de $5120 \times 5120 \mathrm{~m}^{2}$ na horizontal e $2048 \mathrm{~m}$ na vertical. Como condição inicial, foram utilizados perfis de temperatura e umidade de forma a garantir (ou não) similaridade perfeita entre as flutuações dos dois. O escoamento foi forçado por um vento geostrófico $\left(U_{g}, V_{g}\right)=(16,0) \mathrm{ms}^{-1}$.

A heterogeneidade da superfície foi introduzida no modelo na forma de diferentes fluxos superficiais: a direção $x$ foi dividida em patches aos quais foram atribuídos diferentes fluxos (conforme Tabela 1). Como forma de controle do balanço de energia, a soma dos fluxos de calor sensível $(H)$ e latente $(L E)$ ao longo de $x$ foi mantida constante, isto é, $(H+L E)_{\text {patch }_{1}}=(H+L E)_{\text {patch }_{2}}$; os fluxos foram impostos na forma de covariâncias entre as flutuações de velocidade vertical do vento $\left(w^{\prime}\right)$, e temperatura $\left(\theta^{\prime}\right)$ e de umidade específica $\left(q^{\prime}\right)$ em $z=0 \mathrm{~m}$.

O tempo total de simulação foi de 4 horas, com passo de tempo de 0,1 segundos. As simulações foram realizadas utilizando 32 processadores do cluster disponível no Departamento de Meteorologia da Pennsylvania State University nos EUA.

Tabela 1: Número de patches, tipo de perfil inicial de umidade específica (S: umidade aumenta com z; NS: umidade diminui com z), fluxos superficiais de temperatura $\left(\overline{w^{\prime} \theta^{\prime}}\right)$ e umidade específica $\left(\overline{w^{\prime} q^{\prime}}\right)$ impostos como condição de contorno para cada um dos tipos de patches.

\begin{tabular}{|l|c|c|c|c|c|c|}
\hline Simulação & \# patches & $\begin{array}{c}\text { perfil de } \\
\boldsymbol{q}_{0}\end{array}$ & $\overline{\bar{w}^{\prime} \theta_{0}^{\prime}}$ & $\overline{w^{\prime} q_{0}^{\prime}}$ & $\overline{w^{\prime} \theta_{0}^{\prime}}$ & $\overline{w^{\prime} q_{0}^{\prime}}$ \\
$($ patch 1) & patch 1) & patch 2) & patch 2) \\
\hline THET12 & 2 & $\mathrm{~S}$ & 0,15 & 0,002 & 0,05 & 0,006 \\
\hline THET13 & 10 & $\mathrm{~S}$ & 0,15 & 0,002 & 0,05 & 0,006 \\
\hline THET14 & 1 & $\mathrm{~S}$ & 0,10 & 0,004 & 0,10 & 0,004 \\
\hline THET15 & 2 & $\mathrm{~S}$ & 0,17 & 0,001 & 0,025 & 0,007 \\
\hline THET16 & 2 & $\mathrm{~S}$ & 0,11 & 0,0036 & 0,09 & 0,0044 \\
\hline THET17 & 2 & $\mathrm{NS}$ & 0,15 & 0,002 & 0,05 & 0,006 \\
\hline
\end{tabular}

\section{RESULTADOS}

Para todas as simulações, o perfil médio horizontal de $r_{\theta q}$ é apresentado na Fig. 1-a; estes perfis são para o primeiro ponto vertical com saída temporal, isto é, $24 \mathrm{~m}$. Observa-se que, quanto maior a diferença entre os fluxos dos dois tipos de patches - ou quanto mais heterogênea for a superfície - menor é a correlação na CLS. Na Fig. 1-b são apresentados os perfil médios verticias de $r_{\theta q}$ para as 6 simulações. Na região mais próxima à superfície, 
quanto maior a diferença entre os fluxos dos patches tipo 1 e 2 , menor é o coeficiente de correlação, porém podem ser considerados perfeitamente correlacionados acima da chamada altura de mistura; para o caso THET17, $r_{\theta q}$ vai de $\sim 0,9$ para $-1,0$ pois o perfil inicial de umidade específica decresce acima da CLA.

\section{CONSIDERAÇÕES FINAIS}

As análises preliminares dos resultados destas simulações demonstram que quanto maior a diferença entre os fluxos superficiais de cada um dos patches, menor é a correlação entre temperatura e umidade na região da CLS. Acima da CLS os escalares ficam bem próximos da similaridade perfeita. A partir desta análise preliminar é possível concluir que a heterogeneidade da superfície reduz a similaridade entre os escalares na região mais baixa da atmosfera; acima desta região outras causas devem ser consideradas.
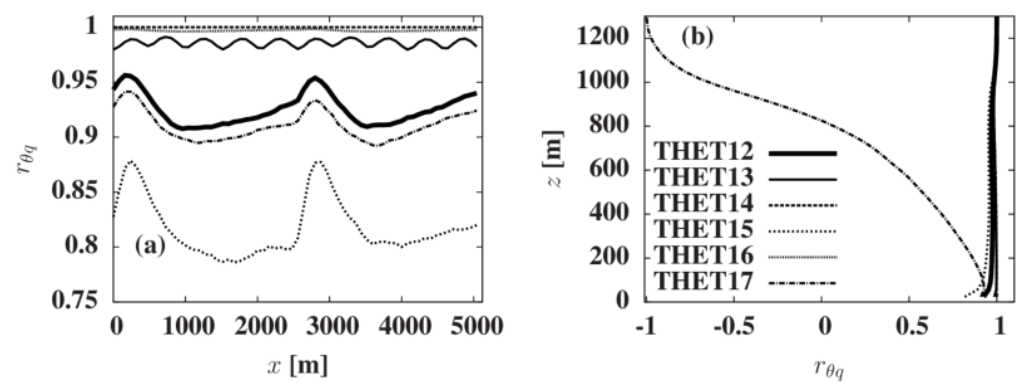

Figura 1: (a) Perfis médios horizontais de $r_{\theta q}$ em $x$ para $z=24 \mathrm{~m}$ para todas as simulações. (b) Perfis médios verticais de $r_{\theta q}$ para as todas as simulações.

\section{Agradecimentos}

Esta pesquisa foi desenvolvida durante o doutorado-sanduíche de Diana Maria Cancelli junto ao Departamento de Meteorologia da Pennsylvania State University com apoio do Programa Ciência sem Fronteiras - CNPq/MCT, processo número 201974/2011-8.

\section{Referências}

CANCELLI, D. M., DIAS, N. L., e CHAMECKI, M. (2012). Dimensionless criteria for the production-dissipation equilibrium of scalar fluctuations and their implications for scalar similarity. Water Resour. Res., 48:W10522.

KUMAR, V., KLEISSL, J., MENEVEAU, C., e PARLANGE, M. B. (2006). Large-eddy simulation of a diurnal cycle of the atmospheric boundary layer: Atmospheric stability and scaling issues. Water Resour. Res., 42:W06D09. 